\title{
A New Algorithm for Distributed Control Problem with Shortest-Distance Constraints
}

\author{
Yu Zhou, ${ }^{1}$ Wenfeng Zheng, ${ }^{1}$ and Zhixi Shen ${ }^{2}$ \\ ${ }^{1}$ School of Automation Engineering, University of Electronic Science and Technology of China, Chengdu 610054, China \\ ${ }^{2}$ Key Laboratory of Dependable Service Computing in Cyber Physical Society, Ministry of Education and School of Automation, \\ Chongqing University, Chongqing 400044, China \\ Correspondence should be addressed to Zhixi Shen; shenzhixi@cqu.edu.cn
}

Received 4 March 2016; Revised 27 May 2016; Accepted 22 November 2016

Academic Editor: Zhan Shu

Copyright (C) 2016 Yu Zhou et al. This is an open access article distributed under the Creative Commons Attribution License, which permits unrestricted use, distribution, and reproduction in any medium, provided the original work is properly cited.

\begin{abstract}
This paper investigates the distributed shortest-distance problem of multiagent systems where agents satisfy the same continuoustime dynamics. The objective of multiagent systems is to find a common point for all agents to minimize the sum of the distances from each agent to its corresponding convex region. A distributed consensus algorithm is proposed based on local information. A sufficient condition also is given to guarantee the consensus. The simulation example shows that the distributed shortest-distance consensus algorithm is effective for our theoretical results.
\end{abstract}

\section{Introduction}

In recent years, distributed control of multiagent systems has attracted considerable attention within control community because of its important applications including distributed task allocation, distributed motion planning, and distributed alignment problems [1-8]. For example, in [1], Nedić et al. introduced a distributed projected consensus algorithm for discrete-time multiagent systems where each agent lies in a closed convex set and gave corresponding convergence analysis on dynamically changing balanced graphs. Founded on the work of [1], [5, 6] considered the networks of fixed and switching topologies. In [7], Matei and Baras proposed the consensus-based multiagent distributed subgradient method to solve the collaborative optimization of an objective function. In [8], Lin and Ren studied the constrained consensus problem in unbalanced networks with communication delays.

In this paper, we will study the distributed control problem with shortest-distance constraints. Distributed shortestdistance consensus problem is one important problem in the distributed control of multiagent systems. The objective of multiagent systems is to find a common point for all agents to minimize the sum of squared distances from each agent to its corresponding convex region. For example, [9] investigated consensus and optimization problems for directed networks of agents with external disturbances. Currently, most of the existing related works concentrate on the case where the intersection set of all convex regions is nonempty [1, 10-12]. A projected consensus algorithm was proposed to solve the constrained consensus problem where each agent is restricted in its own convex set [1]. Reference [10] proposed a class of subgradient-based methods, where some estimate of the optimal solution can be delivered over the network through randomized iteration. In [11], Johansson et al. introduced a subgradient method based on consensus steps to solve coupled optimization problems with fixed undirected topology. In [12], Lou et al. proposed an approximately projected consensus algorithm to achieve the intersection of convex sets. In [13], Wang and Elia proposed a distributed continuous-time algorithm to achieve optimization by controlling the sum of subgradients of convex functions. However, the case where the intersection set of all convex regions is empty is rarely concerned. In [14], the case of no intersection is studied, but the sign functions are used to make the system nonsmooth. Reference [15] investigated a distributed optimization problem and proposed a subgradient projection algorithm for multiagent systems subject to nonidentical constraints and communication delays under local communication. Comparing with $[1,12]$, this paper focuses on the constrained 
problem where all convex regions have no intersection and the undirected graph is connected. Following the work of [14], we investigate the distributed control problem with shortestdistance constraints and propose a new distributed shortestdistance consensus algorithm. By a Lyapunov approach, a sufficient condition is given to make all agents converge to the optimal set of the shortest-distance problem. Finally, we provide a simulation example to show that the distributed shortest-distance consensus algorithm is effective for our theoretical results. Different from [14], we calculate the difference and the Euclidean norm between two different agents and use the ratio of them to replace the sign function. It makes the system smoother than that of [14].

\section{Preliminaries}

2.1. Graph Theory. Let $\mathscr{G}(V, \mathscr{E}, A)$ be an undirected graph of node $n$, where $\mathscr{V}=\left\{s_{1}, \ldots, s_{n}\right\}$ is the set of nodes and $\varepsilon \subseteq$ $\mathscr{V} \times \mathscr{V}$ is the set of edges. The node indexes belong to a finite index set $\mathscr{I}=\{1,2,3, \ldots, n\}$. An edge of $\mathscr{G}$ is denoted by $e_{i j}=$ $\left(s_{i}, s_{j}\right)$, where node $s_{i}$ can obtain information from node $s_{j}$. The weighted adjacency matrix is denoted by $A=\left\{a_{i j}\right\}$, where $a_{i i}=0, a_{i j}=a_{j i}>0$ if and only if $\left(s_{i}, s_{j}\right) \in \varepsilon$ and $s_{i} \neq s_{j}$. Since the graph is considered undirected, the adjacency matrix $A$ is a symmetric nonnegative matrix. The set of neighbors of node $s_{i}$ is denoted by $N_{i}=\left\{s_{j} \in \mathscr{V} ;\left(s_{i}, s_{j}\right) \in \mathscr{E}\right\}$. The in-degree and out-degree of node $s_{i}$ are defined as $d_{i n}\left(s_{i}\right)=\sum_{j=1}^{n} a_{j i}$ and $d_{o}\left(s_{i}\right)=\sum_{j=1}^{n} a_{i j}$. Then, the Laplacian corresponding to the undirected graph is defined as $L=\left[l_{i j}\right]$, where $l_{i j}=d_{o}\left(s_{i}\right)$ and $l_{i j}=-a_{i j}, i \neq j$. Obviously, the Laplacian of any undirected graph is symmetric. A path is a sequence of ordered edges of the form $\left(s_{i 1}, s_{i 2}\right),\left(s_{i 2}, s_{i 3}\right), \ldots$, where $i_{j} \in \mathscr{I}$ and $s_{i j} \in \mathscr{V}$. If there is a path from every node to every other node, the graph is said to be connected [16].

Lemma 1 (see [16]). If the undirected graph $\mathscr{G}$ is connected, then the Laplacian $L$ of $\mathscr{G}$ has the following properties:

(1) Zero is an eigenvalue of $L$, and $\mathbf{1}_{n}$ is the corresponding eigenvector; that is, $L \mathbf{1}_{n}=0$.

(2) The rest $n-1$ eigenvalues are all positive and real.

2.2. Convex Theory. Let $\operatorname{dist}(\bar{x}, X)$ be the standard Euclidean distance of a vector $\bar{x}$ from a set $X$; that is,

$$
\operatorname{dist}(\bar{x}, X)=\inf _{x \in X}\|\bar{x}-x\|
$$

The projection of vector $x$ on a closed convex set $X$ is denoted by the projection term $S_{X}(x)$; that is,

$$
S_{X}(x)=\arg \min _{\bar{x} \in X}\|x-\bar{x}\|,
$$

where $\|x\|$ denotes the standard Euclidean norm, $\|x\|=\sqrt{x^{T} x}$ [17].

Lemma 2 (see [1]). Suppose that $X$ is a nonempty closed convex set in $\mathbb{R}^{m}$, the squared distance function $\rho(x)=$
$(1 / 2)\left\|\left(x-S_{X}(x)\right)\right\|^{2}, x \in \mathbb{R}^{m}$ is continuously differentiable in $x$. Then, we have

$$
\begin{aligned}
\left\|S_{X}(x)-y\right\|^{2} & \leq\|x-y\|^{2}-\left\|S_{X}(x)-x\right\|^{2}, \\
\left\|S_{X}(x)-S_{X}(z)\right\| & \leq\|x-z\| \\
\left(S_{X}(x)-S_{X}(z)\right)^{T}(x-z) & \geq\left\|S_{X}(x)-S_{X}(z)\right\|^{2}, \\
d \frac{1}{2}\left(\left\|x-S_{X}(x)\right\|^{2}\right) & =\left(S_{X}(x)-x\right) d x,
\end{aligned}
$$

where $x, z \in \mathbb{R}^{m}, y \in X$, and $d(\cdot)$ is differential operator.

Lemma 3 (see [18]). LaSalle's invariance principle: consider an autonomous system of the form $\dot{x}=f(x)$, with $f(x)$ continuous, and let $V(x): \mathfrak{R}^{n} \rightarrow \mathfrak{R}$ be a scalar function with continuous first partial derivatives on $\Omega_{l}=\left\{x \in \mathfrak{R}^{n}: V(x) \leq\right.$ l). Assume that

(1) for some $l>0$ the set $\Omega_{l}$ defined by $V(x) \leq l$ is bounded.

(2) $\dot{V}(x) \leq 0$ for all $x$ in $\Omega_{l}$.

If $\Omega_{l}$ extends to the whole space $\mathfrak{R}^{n}$, then global asymptotic stability can be established. Define $R=\left\{x \in \mathfrak{R}^{n}: \dot{V}(x)=0\right\}$. Then if $R$ contains no other trajectories other than $x=0$, then the origin 0 is asymptotically stable. In summary,

(1) if $V(x)$ is a negative semidefinite in a region $\Omega$, where $V(x) \leq 0$, then a solution starting in the interior of $\Omega$ remains there;

(2) if, in addition, no solutions (except the equilibrium $x=$ 0 ) remain in $R$ (the subset of $\Omega$ where $\dot{V}(x)=0$ ), then all solutions starting in the interior of $\Omega$ will converge to the equilibrium.

Lemma 4 (see [19]). Let $f_{0}(\chi): \mathbb{R}^{m} \rightarrow \mathbb{R}$ be a differentiable convex function. $f_{0}(\chi)$ is minimized if and only if $d f_{0}(\chi) / d \chi=$ 0 .

\section{Problem Description and Results}

3.1. Problem Description. The multiagent system under consideration contains $n$ agents, where each agent corresponds to a certain bounded convex set, denoted by $X_{i}$. Our objective is to design a distributed consensus algorithm for the system to make all agents able to reach consensus and minimize the sum of squared distances between the global point and the convex sets.

$$
\frac{1}{2} \sum_{i=1}^{n}\left\|\left(x-S_{X_{i}}(x)\right)\right\|^{2}, \quad X_{i} \in \mathbb{R}^{m} .
$$

We assume that the closed set $X_{i} \in \mathbb{R}^{m}$ is nonempty, $\mathbb{R}^{m}$ is the set of all dimensional real column vectors, and $\bigcap_{i=1}^{n} X_{i}=\emptyset$. In other words, we need to find a global optimal point that minimizes the sum of squared distances form $i$ to its all closed convex sets. Each agent is assumed to have the following continuous-time dynamics:

$$
\dot{x}_{i}(t)=u_{i}(t), \quad i \in \mathscr{I},
$$


where $x_{i} \in \mathbb{R}^{m}$ is the state of the $i$ th agent, $\mathscr{F}$ is the index set $\{1,2, \ldots, n\}$, and $u_{i}(t)$ is the control input of the $i$ th agent.

3.2. A New Distributed Shortest-Distance Consensus Algorithm. We propose a new distributed shortest-distance consensus algorithm as the following form:

$$
u_{i}(t)=\alpha \sum_{j \in N_{i}(t)} \frac{x_{j}(t)-x_{i}(t)}{\left\|x_{j}(t)-x_{i}(t)\right\|}+S_{X_{i}}\left(x_{i}(t)\right)-x_{i}(t),
$$

$$
i \in \mathscr{I}
$$

where $\alpha>0$ is a positive integer and $\mathscr{I}$ is the index set $\{1,2$, $\ldots, n\}$ and $x_{j}(t) \neq x_{i}(t) . N_{i}(t)$ is neighbor set of agent $i$ at time $t$, and $x_{i}(t)$ is the state of agent $i$ at the time $t$. If $x_{j}(t)=x_{i}(t)$, we define $\sum_{j \in N_{i}(t)}\left(\left(x_{j}(t)-x_{i}(t)\right) /\left\|x_{j}(t)-x_{i}(t)\right\|\right)=0$.

Assumption 5. Suppose that, for $i$, the closed set $X_{i}$ is compact set; that is,

$$
\|x\| \leq g, \quad g>0,
$$

where $i \in \mathscr{I}$ and $x \in X_{i}$.

Assumption 6. Suppose that the set $X_{i} \in \mathbb{R}^{m}$ is nonempty and the set $X_{i}$ satisfies

$$
\bigcap_{i=1}^{n} X_{i}=\emptyset
$$

Theorem 7. Suppose that the undirected graph $\mathscr{G}$ is connected. Under Assumption 6, if $\alpha>2 n g$, using Lemmas 2 and 3 for (4), the convex function (4) is minimized as $t \rightarrow+\infty$.

Proof. Define a Lyapunov function

$$
V_{a}(t)=\frac{1}{2} \sum_{i=1}^{n}\left\|x_{i}(t)-\frac{1}{n} \sum_{j=1}^{n} x_{j}(t)\right\|^{2} .
$$

According to $d(1 / 2)\left\|x_{i}-x_{j}\right\|^{2} / d x=\left(x_{i}-x_{j}\right)^{T}\left(\dot{x}_{i}-\dot{x}_{j}\right)$ and (5)-(6), we get

$$
\begin{aligned}
\dot{V}_{a}(t) & =\sum_{i=1}^{n}\left[x_{i}(t)-\frac{1}{n} \sum_{j=1}^{n} x_{j}(t)\right]^{T} \dot{x}_{i}(t) \\
= & \sum_{i=1}^{n}\left[x_{i}(t)-\frac{1}{n} \sum_{j=1}^{n} x_{j}(t)\right]^{T} \\
\cdot & {\left[\alpha \sum_{j \in N_{i}(t)} \frac{x_{j}(t)-x_{i}(t)}{\left\|x_{j}(t)-x_{i}(t)\right\|}+S_{X_{i}}\left(x_{i}(t)\right)-x_{i}(t)\right] . }
\end{aligned}
$$

Suppose that the undirected graph $\mathscr{G}$ is connected. It follows that

$$
\begin{aligned}
& \dot{V}_{a}(t) \\
& =\sum_{i=1}^{n} \sum_{j \in N_{i(t)}} \frac{\alpha}{2}\left[x_{i}(t)-x_{j}(t)\right]^{T} \frac{x_{j}(t)-x_{i}(t)}{\left\|x_{j}(t)-x_{i}(t)\right\|} \\
& \quad+\sum_{i=1}^{n}\left[x_{i}(t)-\frac{1}{n} \sum_{j=1}^{n} x_{j}(t)\right]^{T} S_{X_{i}}\left(x_{i}(t)\right) \\
& \quad-\sum_{i=1}^{n}\left[x_{i}(t)-\frac{1}{n} \sum_{j=1}^{n} x_{j}(t)\right]^{T}\left[x_{i}(t)-\frac{1}{n} \sum_{j=1}^{n} x_{j}(t)\right] \\
& =\sum_{i=1}^{n} \sum_{j \in N_{i(t)}} \frac{\alpha}{2}\left[x_{i}(t)-x_{j}(t)\right]^{T} \frac{x_{j}(t)-x_{i}(t)}{\left\|x_{j}(t)-x_{i}(t)\right\|} \\
& \quad+\sum_{i=1}^{n}\left[x_{i}(t)-\frac{1}{n} \sum_{j=1}^{n} x_{j}(t)\right]^{T} S_{X_{i}}\left(x_{i}(t)\right) \\
& \quad-\sum_{i=1}^{n}\left\|x_{i}(t)-\frac{1}{n} \sum_{j=1}^{n} x_{j}(t)\right\|^{2} .
\end{aligned}
$$

Let $\left\|x_{i_{m}}(t)-x_{j_{m}}(t)\right\|$ denote the maximum distance between agent $i$ and agent $j$ at time $t$; that is,

$$
\left\|x_{i_{m}}(t)-x_{j_{m}}(t)\right\|=\max _{i, j \in \mathcal{I}}\left\|x_{i}(t)-x_{j}(t)\right\| .
$$

From $i_{m}, j_{m} \in \mathscr{I}$, we get

$$
\left\|x_{i}(t)-\frac{1}{n} \sum_{j=1}^{n} x_{j}(t)\right\| \leq\left\|x_{i_{m}}(t)-x_{j_{m}}(t)\right\| .
$$

Since undirected graph $\mathscr{G}(V, \mathscr{E}, A)$ is connected, the distance between node $v_{i_{m}}$ and node $v_{j_{m}}$ is less than the sum of squared distances between agent $i$ and its neighbors; that is,

$$
\begin{aligned}
& \left\|x_{i_{m}}(t)-x_{j_{m}}(t)\right\| \leq \sum_{i=1}^{n} \sum_{j \in N_{i}(t)}\left\|x_{i}(t)-x_{j}(t)\right\|, \\
& \left\|x_{i}(t)-x_{j}(t)\right\| \leq-\left[x_{i}(t)-x_{j}(t)\right]^{T} \frac{x_{j}(t)-x_{i}(t)}{\left\|x_{j}(t)-x_{i}(t)\right\|} .
\end{aligned}
$$

Combining (13)-(15), we have

$$
\begin{aligned}
& \left\|x_{i}(t)-\frac{1}{n} \sum_{j=1}^{n} x_{j}(t)\right\| \leq\left\|x_{i_{m}}(t)-x_{j_{m}}(t)\right\| \\
& \quad \leq-\sum_{i=1}^{n} \sum_{j \in N_{i(t)}}\left[x_{i}(t)-x_{j}(t)\right]^{T} \frac{x_{j}(t)-x_{i}(t)}{\left\|x_{j}(t)-x_{i}(t)\right\|} .
\end{aligned}
$$


If $\alpha>2 n g$, substituting (7) and (11), we have

$$
\begin{aligned}
\dot{V}_{a}(t)= & -\sum_{i=1}^{n} \sum_{j \in N_{i(t)}} \frac{\alpha}{2}\left\|x_{i}(t)-x_{j}(t)\right\| \\
& +\sum_{i=1}^{n}\left[x_{i}(t)-\frac{1}{n} \sum_{j=1}^{n} x_{j}(t)\right]^{T} S_{X_{i}}\left(x_{i}(t)\right) \\
& -\sum_{i=1}^{n}\left\|x_{i}(t)-\frac{1}{n} \sum_{j=1}^{n} x_{j}(t)\right\| \|^{2} \\
\leq & -\sum_{i=1}^{n} \sum_{j \in N_{i(t)}}\left\|x_{i}(t)-x_{j}(t)\right\|\left(\frac{\alpha}{2}-n g\right) \\
& -\sum_{i=1}^{n}\left\|x_{i}(t)-\frac{1}{n} \sum_{j=1}^{n} x_{j}(t)\right\| \|^{2} \\
\leq & -\sum_{i=1}^{n} \sum_{j \in N_{i(t)}}\left\|x_{i}(t)-x_{j}(t)\right\|\left(\frac{\alpha}{2}-n g\right) .
\end{aligned}
$$

From (14),

$$
\begin{aligned}
& \sum_{i=1}^{n} \sum_{j \in N_{i(t)}}\left\|x_{i}(t)-x_{j}(t)\right\| \geq\left\|x_{i_{m}}(t)-x_{j_{m}}(t)\right\| \\
& \quad \geq \sqrt{\frac{2 V_{a}(t)}{n}} .
\end{aligned}
$$

Thus

$$
\dot{V}_{a}(t) \leq-\sqrt{\frac{2 V_{a}(t)}{n}} .
$$

That is, $\dot{V}_{a}(t) / \sqrt{V_{a}(t)} \leq-\sqrt{2 / n}$. And integrating both sides of this inequality from 0 to $t$, we have

$$
\sqrt{V_{a}(+\infty)}-\sqrt{V_{a}(0)} \leq-\sqrt{\frac{2}{n}} t .
$$

Since $V_{a}(t) \geq 0$, it is clear that $V_{a}(t)$ vanishes to zero in finite time and hence all agents reach a consensus in finite time. Thus, there is a constant $T>0$ such that $V_{a}(t)=0$ and $x_{i}(t)=$ $x_{j}(t)$ for all $i, j \in \mathscr{I}$ and all $t>T$. Thus

$$
\lim _{t \rightarrow+\infty}\left(x_{j}(t)-x_{i}(t)\right)=0
$$

Consider the average value of all agents, denoted by $x^{*}(t)=(1 / n) \sum_{i=1}^{n} x_{i}(t)$, for $t>T$. It is clear that

$$
\dot{x}^{*}(t)=\frac{1}{n} \sum_{k=1}^{n} S_{X_{k}}\left(x^{*}(t)\right)-x^{*}(t)
$$

for all $t>T$. Consider the Lyapunov function

$$
V_{b}(t)=\frac{1}{2} \sum_{i=1}^{n}\left\|x^{*}(t)-S_{X_{i}}\left(x^{*}(t)\right)\right\|^{2} \leq 0
$$

for $t>T$.

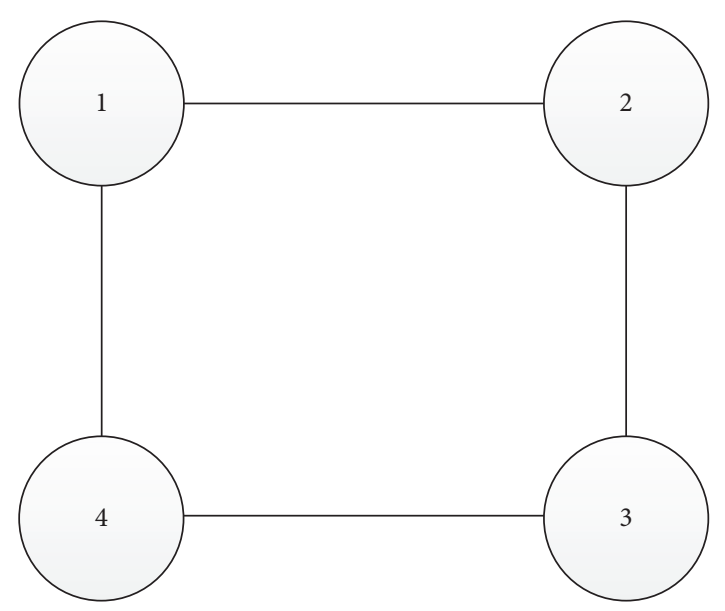

FIGURE 1: Topology.

Calculating $\dot{V}_{b}(t)$, for all $t>T$, we have

$$
\begin{aligned}
\dot{V}_{b}(t) & =\sum_{i=1}^{n}\left[x^{*}(t)-S_{X_{i}}\left(x^{*}(t)\right)\right]^{T} \\
\cdot & {\left[\frac{1}{n} \sum_{k=1}^{n} S_{X_{k}}\left(x^{*}(t)\right)-x^{*}(t)\right] } \\
= & -\frac{1}{n}\left\|\sum_{i=1}^{n}\left(x^{*}(t)-S_{X_{i}}\left(x^{*}(t)\right)\right)\right\|^{2} .
\end{aligned}
$$

Since each $X_{i}$ is bounded and $\dot{V}_{b}(t) \leq 0$, thus $V_{b}(t)$ is bounded. And from Lemma $2,(1 / n) \sum_{k=1}^{n} S_{X_{k}}\left(x^{*}(t)\right)-x^{*}(t)$ is continuous. Note that $V_{b}(t) \leq 0$; then from Lemma 3 , we have $x^{*}(t)$ converges to the set $\left\{\dot{V}_{b}(t)=0\right\}$ as $t \rightarrow+\infty . \dot{V}_{b}(t)=0$ implies that $(1 / n) \sum_{k=1}^{n} S_{X_{k}}\left(x^{*}(t)\right)-x^{*}(t)=0$. Thus

$$
\lim _{t \rightarrow+\infty}\left(\frac{1}{n} \sum_{k=1}^{n} S_{X_{k}}\left(x^{*}(t)\right)-x^{*}(t)\right)=0 .
$$

Note that $(d / d t)(1 / 2) \sum_{i=1}^{n}\left\|\left(x^{*}(t)-S_{X_{i}}\left(x^{*}(t)\right)\right)\right\|^{2}=x^{*}(t)-$ $(1 / n) \sum_{k=1}^{n} S_{X_{k}}\left(x^{*}(t)\right)$. From Lemma 4 and (25), the convex function (4) is minimized as $t \rightarrow+\infty$.

Remark 8. In Theorem 7, we only discuss the undirected connected graphs and our future work will be directed to the general jointly connected graphs.

\section{Simulation}

In this section, we provide a numerical example to show the effectiveness of Theorem 7. We use the fixed topology which is showed in Figure 1. And let $\alpha=10, X_{1}=\left\{\left(x_{11}, x_{12}\right)^{T} \in \mathbb{R}^{2} \mid\right.$ $\left.\left\|\left(x_{11}, x_{12}\right)^{T}\right\| \leq 5\right\}, X_{2}=\left\{\left(x_{21}, x_{22}\right)^{T} \in \mathbb{R}^{2} \mid \|\left(x_{21}, x_{22}\right)^{T}-\right.$ $\left.(-10,30)^{T} \| \leq 2.5\right\}, X_{3}=\left\{\left(x_{31}, x_{32}\right)^{T} \in \mathbb{R}^{2} \mid \|\left(x_{31}, x_{32}\right)^{T}-\right.$ $\left.(20,10)^{T} \| \leq 2.5\right\}$, and $X_{4}=\left\{\left(x_{41}, x_{42}\right)^{T} \in \mathbb{R}^{2} \mid \|\left(x_{41}, x_{42}\right)^{T}-\right.$ $\left.(30,30)^{T} \| \leq 2.5\right\}$. Figure 2 shows the simulation results. 


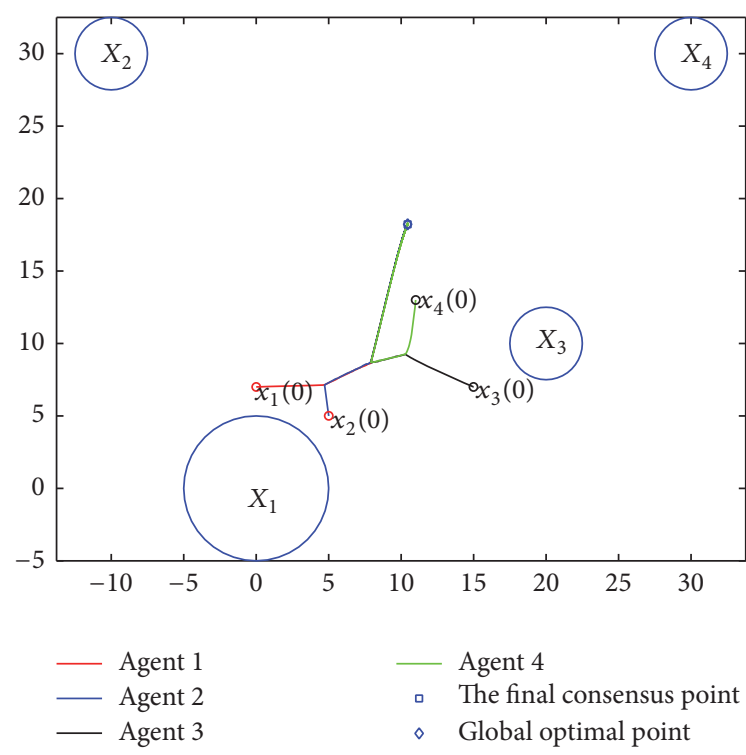

FIGURE 2: State trajectories of agents 1, 2, 3, and 4.

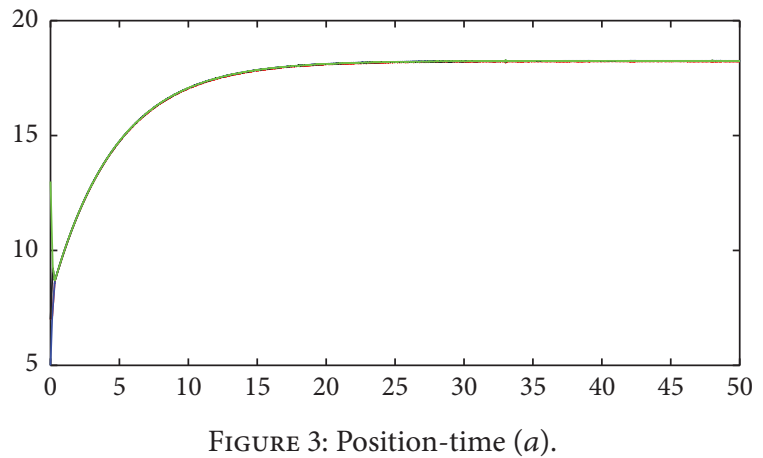

Figures 3 and 4 show the position trajectories, respectively. It is obvious that all agents reach a consensus point that is the optimal point of the function (4). This is consistent with Theorem 7.

\section{Conclusion}

In the paper, we purpose a new distributed shortest-distance algorithm for multiagent systems. The objective of multiagent systems is to find a common point for all agents to minimize the sum of squared distances from each agent to its corresponding convex regions. A sufficient condition also is given to guarantee the consensus. And the simulation example is given to show that the distributed shortestdistance consensus algorithm is effective for our theoretical results.

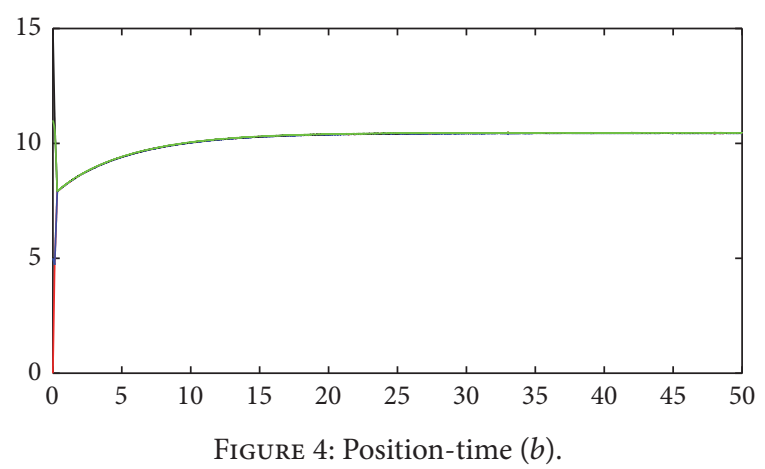

\section{Competing Interests}

The authors declare that there is no conflict of interests regarding the publication of this paper.

\section{References}

[1] A. Nedić, A. Ozdaglar, and P. A. Parrilo, "Constrained consensus and optimization in multi-agent networks," Transactions on Automatic Control, vol. 55, no. 4, pp. 922-938, 2010.

[2] C. Duan, J. Cleland-Huang, and B. Mobasher, "A consensus based approach to constrained clustering of software requirements," in Proceedings of the ACM Conference on Information and Knowledge Management (CIKM '08), pp. 1073-1082, Napa Valley, Calif, USA, October 2008.

[3] U. Lee and M. Mesbahi, "Constrained consensus via logarithmic barrier functions," in Proceedings of the 50th IEEE Conference on Decision and Control and European Control Conference (CDCECC '11), pp. 3608-3613, December 2011.

[4] S. S. Ram, A. Nedić, and V. V. Veeravalli, "Incremental stochastic subgradient algorithms for convex optimization," SIAM Journal on Optimization, vol. 20, no. 2, pp. 691-717, 2009.

[5] R. Olfati-Saber and R. M. Murray, "Consensus problems in networks of agents with switching topology and time-delays," Transactions on Automatic Control, vol. 49, no. 9, pp. 1520-1533, 2004.

[6] M. Zhu and S. Martínez, "An approximate dual subgradient algorithm for multi-agent non-convex optimization," IEEE Transactions on Automatic Control, vol. 58, no. 6, pp. 1534-1539, 2013.

[7] I. Matei and J. S. Baras, "Performance evaluation of the consensus-based distributed subgradient method under random communication topologies," IEEE Journal on Selected Topics in Signal Processing, vol. 5, no. 4, pp. 754-771, 2011.

[8] P. Lin and W. Ren, "Constrained consensus in unbalanced networks with communication delays," Institute of Electrical and Electronics Engineers. Transactions on Automatic Control, vol. 59, no. 3, pp. 775-781, 2014.

[9] P. Lin, Y. Jia, and L. Li, "Distributed robust Ho consensus control in directed networks of agents with time-delay," Systems \& Control Letters, vol. 57, no. 8, pp. 643-653, 2008.

[10] A. Nedić and A. Ozdaglar, "Distributed subgradient methods for multi-agent optimization," Transactions on Automatic Control, vol. 54, no. 1, pp. 48-61, 2009.

[11] B. Johansson, T. Keviczky, M. Johansson, and K. H. Johansson, "Subgradient methods and consensus algorithms for solving convex optimization problems," in Proceedings of the 47th IEEE 
Conference on Decision and Control (CDC '08), pp. 4185-4190, IEEE, Cancun, Mexico, December 2008.

[12] Y. Lou, G. Shi, K. H. Johansson, and Y. Hong, "Reaching optimal consensus for multi-agent systems based on approximate projection," in Proceedings of the 10th World Congress on Intelligent Control and Automation (WCICA '12), pp. 2794-2800, July 2012.

[13] J. Wang and N. Elia, "Control approach to distributed optimization," in Proceedings of the 48th Annual Allerton Conference on Communication, Control, and Computing (Allerton '10), pp. 557561, Monticello, Va, USA, October 2010.

[14] P. Lin, W. Ren, and J. A. Farrel, "Distributed continuoustime optimization: nonuniform gradient gains, finite-time convergence, and convex constraint set," IEEE Transactions on Automatic Control, 2016.

[15] P. Lin, W. Ren, and Y. Song, "Distributed multi-agent optimization subject to nonidentical constraints and communication delays," Automatica. A Journal of IFAC, the International Federation of Automatic Control, vol. 65, pp. 120-131, 2016.

[16] N. Biggs, Algebraic graph theory, Cambridge University Press, London, UK, 1974.

[17] D. P. Bertsekas, Convex optimization theory, Athena Scientific, Nashua, NH, USA, 2009.

[18] F. Blanchini, "Set invariance in control," Automatica, vol. 35, no. 11, pp. 1747-1767, 1999.

[19] S. Boyd and L. Vandenberghe, Convex Optimization, Cambridge University Press, 2004. 


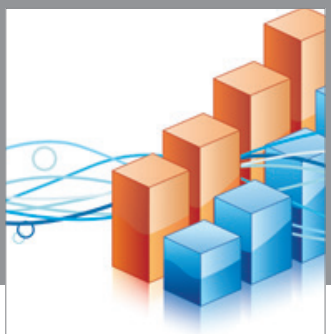

Advances in

Operations Research

vatem alat4

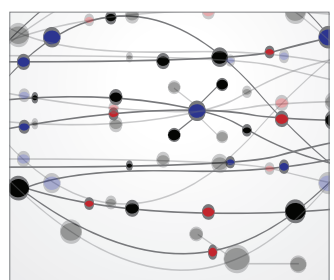

\section{The Scientific} World Journal
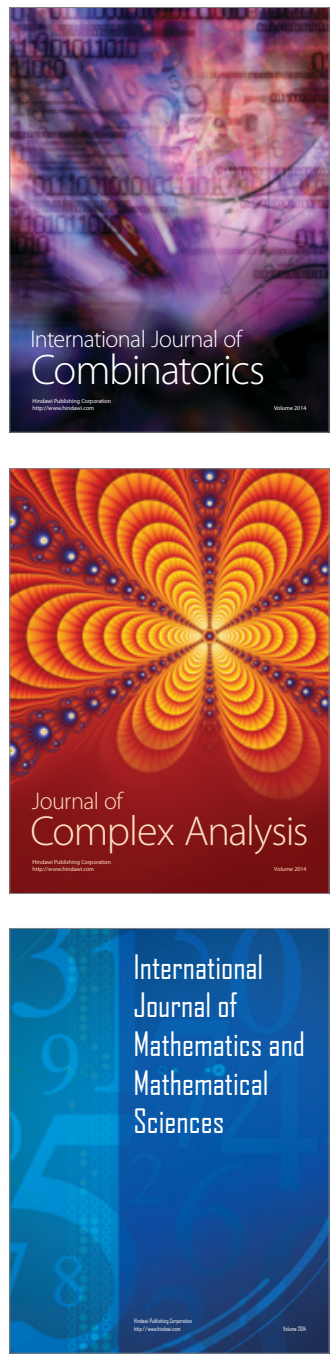
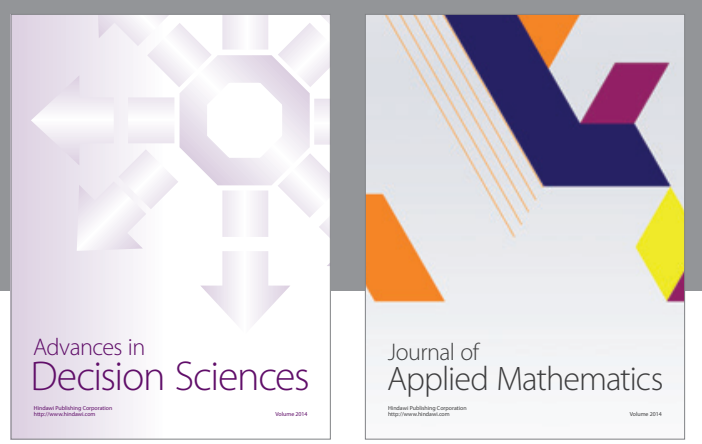

Algebra

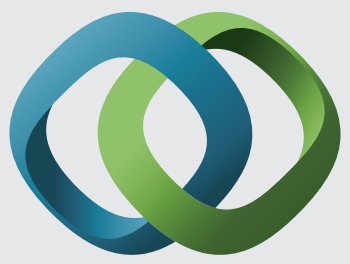

\section{Hindawi}

Submit your manuscripts at

http://www.hindawi.com
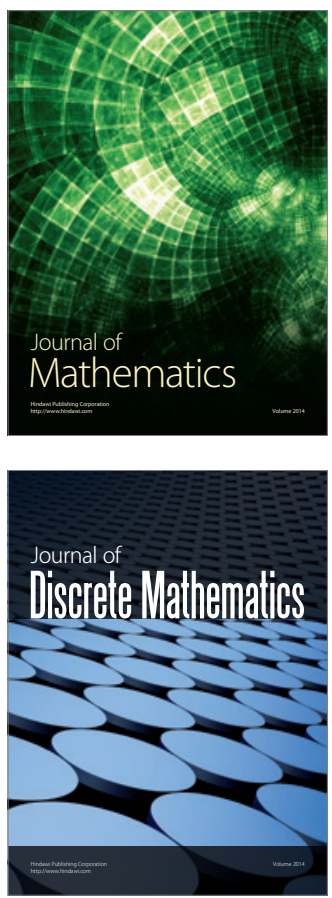

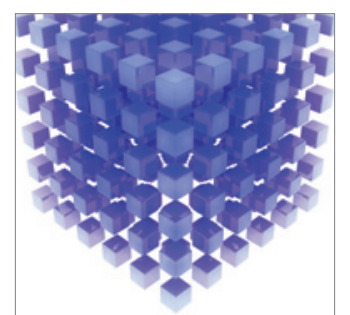

Mathematical Problems in Engineering
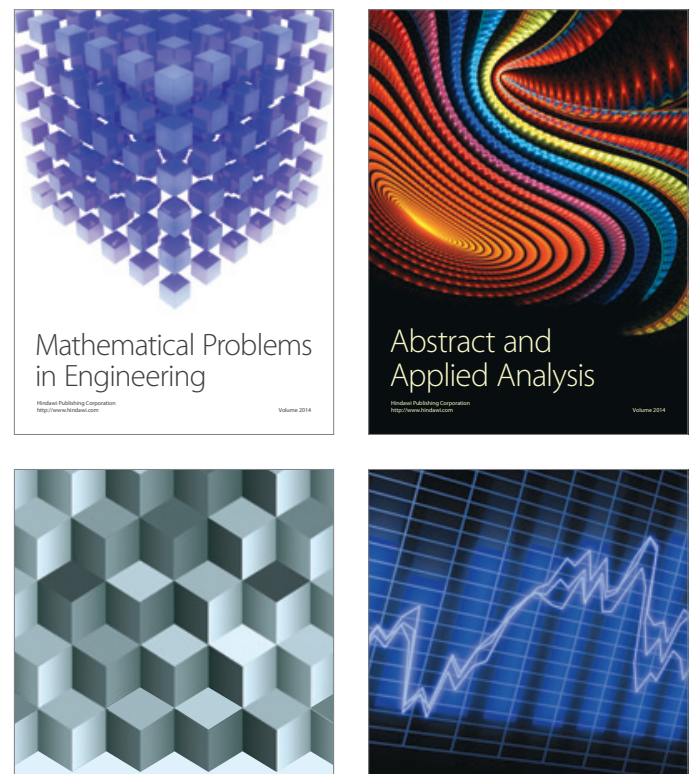

Journal of

Function Spaces

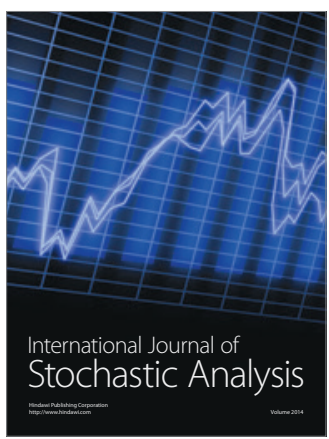

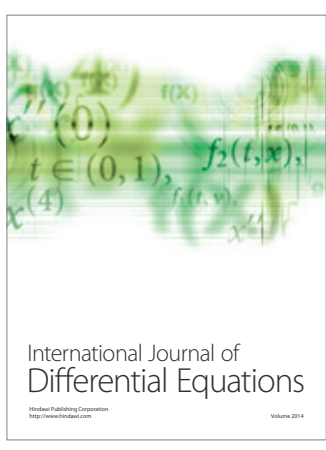
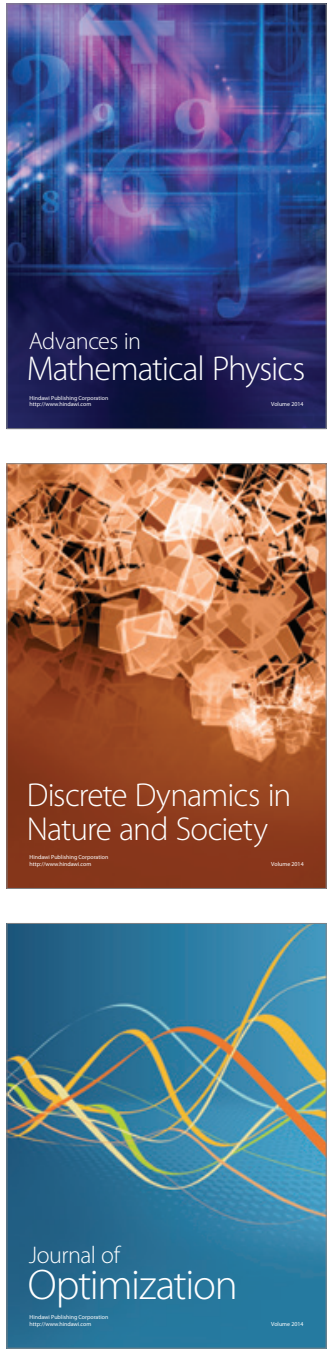